\title{
Inefficiency as the major driver of excess costs in lung resection
}

\author{
Donald E. Fry, MD, ${ }^{\mathrm{a}, \mathrm{b}, \mathrm{c}}$ Michael Pine, MD, MBA, ${ }^{\mathrm{a}, \mathrm{d}}$ Barbara L. Jones, MA, ${ }^{\mathrm{a}}$ and Roger J. Meimban, $\mathrm{PhD}^{\mathrm{a}}$
}

\begin{abstract}
Background: Risk-adjusted outcomes of surgical care are important for quality and cost assessments. Although cardiac surgery is commonly studied, risk-adjusted analysis of excess costs of lung resection has not been pursued.
\end{abstract}

\begin{abstract}
Methods: We used 2002 to 2005 National Inpatient Sample of the Healthcare Cost and Utilization Project data to evaluate adverse outcomes and costs in elective lung resections in hospitals with more than 20 cases during that period. Adverse outcomes were inpatient death or excessive risk-adjusted postoperative stay. Logistic models were defined to predict adverse outcomes. Linear models were designed to predict costs. Hospitalspecific adverse outcome rates and costs were measured to define performance outliers. Cost-effective reference hospitals were used to define total excess costs.
\end{abstract}

Results: Among 12,182 patients at 215 hospitals undergoing lung resection, there were 336 inpatient deaths $(2.8 \%)$ and 880 live discharges with prolonged risk-adjusted postoperative stay $(7.2 \%)$. Predictive models for mortality and risk-adjusted postoperative stay had $\mathrm{C}$ statistics of 0.773 and 0.643 , respectively. There were 11 ineffective hospitals $(5.1 \%)$ with excessive adverse outcomes $(P<.005)$ and 34 inefficient hospitals $(15.8 \%)$ meeting quality measures but with higher than predicted costs $(P<.0005)$. Ineffective hospitals had costs $\$ 1020$ per case lower than predicted. Inefficient hospitals had costs $\$ 9978$ higher than predicted.

Conclusions: Inefficiency is the major factor in excess inpatient costs associated with lung resection in this model. Although refinements in databases, including total physician costs and postdischarge adverse event costs, will alter models, excess costs of lung resection appear to be driven by inefficiency, not adverse outcomes. (J Thorac Cardiovasc Surg 2011;142:1418-22)

Improvement in the quality of surgical care has been a continuing goal for all surgeons and has recently become the focus of attention by governmental and regulatory agencies. Not only is reduced patient morbidity desirable as a goal in itself, but complications of major operations have been identified as important sources of excess costs. ${ }^{1}$ Reduction of surgical complications can be an important component in efforts to contain health care costs.

The Surgical Care Improvement Project has been a high visibility government initiative focused on the reduction of complications and associated costs related to surgical site infections, postoperatively myocardial events, deep venous thrombophlebitis and pulmonary embolism, and postoperative pulmonary failure. ${ }^{2}$ In its initial design of quality improvement process measures, the Surgical Care

\footnotetext{
From Michael Pine and Associates, ${ }^{\mathrm{a}}$ Chicago, Ill; the Department of Surgery, ${ }^{\mathrm{b}}$ Northwestern University Feinberg School of Medicine, Chicago, Ill; the Department of Surgery, ${ }^{\mathrm{c}}$ University of New Mexico School of Medicine, Albuquerque, NM; and the Department of Medicine, ${ }^{\mathrm{d}}$ University of Chicago School of Medicine, Chicago, Ill.

Disclosures: Authors have nothing to disclose with regard to commercial support. Received for publication May 26, 2011; revisions received July 29, 2011; accepted for publication Aug 25, 2011; available ahead of print Sept 28, 2011.

Address for reprints: Donald E. Fry, MD, Executive Vice President, Michael Pine and Associates, 5020 S Lake Shore Dr, No. 304N, Chicago, IL 60615 (E-mail: dfry@ consultmpa.com).

$0022-5223 / \$ 36.00$

Copyright $($ c 2011 by The American Association for Thoracic Surgery doi: 10.1016/j.jtcvs.2011.08.033
}

Improvement Project targeted high-volume, high-cost procedures of coronary artery bypass grafting, total hip and knee replacement, hysterectomy, peripheral vascular surgery, and colon resection. ${ }^{3}$ Lung resection has not been a focus of the Surgical Care Improvement Project to this point in time.

Our group has analyzed results of major surgical procedures to characterize the relationship between currently achievable improvements in quality and those in reductions in the cost of surgical care. The objective of these analyses has been to identify reference hospitals that meet analytically derived quality performance and efficiency standards. These reference hospitals can be used to calibrate episodebased, risk-adjusted payment models designed to reward hospitals and surgeons financially for delivering costeffective surgical care.

Both the Society of Thoracic Surgery ${ }^{4}$ and the European Society of Thoracic Surgery ${ }^{5}$ have demonstrated considerable interest in improving the quality of surgical care for patients undergoing lung resections. Currently, mortality and morbidity vary substantially among hospitals; however, there is little information available about associated variations in costs. This study applies methods that distinguish between the costs of complications (ie, ineffective care) and routine costs (ie, clinical efficiency). We hypothesize that potential savings and improved quality in lung resections can be identified through comparisons with costeffective reference hospitals. 


\section{Abbreviation and Acronym \\ ICD- = International Classification of Diseases, 9-CM Ninth Revision, Clinical Modification}

\section{MATERIALS AND METHODS}

We studied elective lung resection in patients with International Classification of Diseases, Ninth Revision, Clinical Modification (ICD-9-CM) codes of 32.3 through 32.5 and 32.9. These were selected from the years 2002 to 2005 with the National Inpatient Sample of the Healthcare Cost and Utilization project supplied by the Agency for Healthcare Research and Quality. ${ }^{6}$ Only elective operations performed within 2 days of admission at hospitals with 20 or more cases during the study period were included in the analysis. Patients at hospitals that failed to pass previously described data quality screens ${ }^{7}$ were excluded from the study.

Inpatient mortality and prolonged postoperative risk-adjusted stays for live discharges were classified as adverse outcomes. The hospital cost for each surgical case was computed from total billed charges and the hospital's cost-to-charge ratio. ${ }^{8}$ Costs were adjusted to 2005 dollars. Predicted values were adjusted by hospital-specific wage indices and by regional cost characteristics of urban versus rural hospitals.

A list of ICD-9-CM diagnosis codes for generic and procedure-specific complications was created. Risk factors for possible inclusion in predictive models for patient outcomes were crafted from demographic, diagnostic, and procedural hospital discharge data (Table 1). The generic set of diagnostic risk factors developed by Elixhauser and colleagus ${ }^{9}$ was modified and expanded. Dummy variables for lobectomy and for pneumonectomy were created to account for potentially greater morbidity and cost associated with these procedures. Hospital dummy variables were used during variable selection and model calibration to minimize hospital-specific influences on the final predictive equations. ${ }^{10}$

All analyses were performed with SAS statistical software (version 9.1.3; SAS Institute, Inc, Cary, NC).

\section{Prolonged Risk-Adjusted Postoperative Stay and Adverse Outcomes}

Forward stepwise linear regression was used to derive a predictive model for routine postoperative stay for live discharges without ICD-9$\mathrm{CM}$-coded complications. Risk-adjusted postoperative stay was computed as the difference between the observed and the predicted postoperative stay for each live discharge.

Hospital-specific XmR control charts were used to identify live discharges with risk-adjusted postoperative stay longer than a 3-SD upper control limit (ie, outliers). ${ }^{11,12}$ Outliers were eliminated from each hospital's reference database, and a new 3-SD upper control limit was derived. This process was repeated until no case remaining in a reference database had a risk-adjusted postoperative stay that exceeded the 3-SD upper threshold. Outliers were classified as having had prolonged risk-adjusted postoperative stay; other live discharges were classified as routine cases.

Forward stepwise logistic regression was used to derive predictive equations for inpatient mortality for all discharges and for prolonged riskadjusted postoperative stay for live discharges. The intercepts of these equations were recalibrated so that total observed and predicted values were equal after hospital dummy variables had been removed. The predicted probability of an adverse outcome (ie, death or a prolonged riskadjusted postoperative stay) for each case was computed as $p\{$ death $\}+$ $[(1-p\{$ death $\}) \cdot p$ \{prolonged risk-adjusted postoperative stay $\}]$, where $\mathrm{p}\{$ death $\}$ is the probability of death and $\mathrm{p}\{$ prolonged risk-adjusted postoperative stay\} is the probability of a prolonged risk-adjusted postoperative stay. C statistics were used to determine the discriminatory power of the final predictive equations.

\section{Hospital Costs}

Forward stepwise linear regression was used to derive a predictive model for routine hospital costs on the basis of data for all routine cases. A lower limit for routine hospital costs was derived by computing each hospital's 10th percentile cost and then setting this lower limit equal to the 10th percentile of these costs at all hospitals. This is a method that has previously been used to prevent cases with extremely low cost from adversely influencing the intercept of the cost model. ${ }^{13-15}$ Estimated routine costs for cases with adverse outcomes were computed by the following formula: Estimated costs (adverse outcomes) = Predicted routine cost (model) - (observed routine costs of reference hospital)/(predicted costs of reference hospital). Use of this formula permitted adjustment of the raw calculation from the coefficients of the national model to the pattern of expected routine costs for the reference hospital from which the adverse outcome care originated.

Excess hospital costs attributable to adverse outcomes were computed by subtracting estimated routine costs from actual hospital costs for cases with adverse outcomes. When computed excess hospital costs were negative, they were set to zero. When the computed excess hospital cost for a case was 10 times greater than the corresponding predicted routine cost, the excess cost was set to 10 times the predicted routine cost. Costs that exceeded this upper threshold were assigned to a stop-loss pool. This 10-fold threshold was arbitrarily chosen because it was indexed to predicted costs and consistently has involved only a small number of cases that consisted of a small total cost $(1 \%)$ of the whole population but represents a truly catastrophic cost for the incident hospital. ${ }^{13-15}$

Forward stepwise linear regression was used to derive a predictive model for excess hospital costs of adverse outcomes on the basis of data for all cases with adverse outcomes. A dummy variable for inpatient mortality was added to the set of candidate risk factors. For each case, the predicted cost of a possible adverse outcome was computed as the sum of the probability of dying times the predicted excess cost associated with inpatient mortality and the probability of being discharged alive with a prolonged risk-adjusted postoperative stay multiplied by the predicted excess cost associated with a prolonged risk-adjusted postoperative stay. Each hospital's predicted costs were adjusted to reflect regional wage indices and the hospital's urban or rural location. To determine the explanatory power of the final predictive equations, $r^{2}$ statistics were used.

\section{Cost-Effective Reference Hospitals}

To identify ineffective hospitals with significantly higher-than-average adverse outcome rates, predicted adverse outcome rates were standardized so that the total predicted adverse outcomes equaled the total observed adverse outcomes for the study population. Outlier hospitals whose observed adverse outcome rates exceeded their predicted adverse outcome rates by more than $2.56 \mathrm{SD}(P<.005)$ were classified as ineffective and removed from the reference data set. This arbitrary level was chosen because at most 1 or 2 hospitals would be expected by chance alone to exceed this threshold. Predicted adverse outcome rates for the remaining hospitals were recalculated so that the total predicted and observed rates were equal, and any newly identified outlier hospitals were removed from the reference data set. This process was repeated until no further outlier hospitals could be identified.

To identify inefficient hospitals with substantially higher than average costs, predicted costs for routine cases at effective hospitals (ie, hospitals remaining in the reference data set after ineffective hospitals were removed) were standardized so that total predicted costs equaled total observed costs. Outlier hospitals whose observed per case routine costs were more than twice their predicted per case routine costs and whose observed routine costs exceeded their predicted routine costs by more than 3.29 SD $(P<.0005)$ were classified as inefficient and removed from the reference data set. Because costs are continuous variables and relatively small differences can assume statistical significance, a more extreme threshold was chosen for inefficient outliers than for ineffective ones. Predicted per 


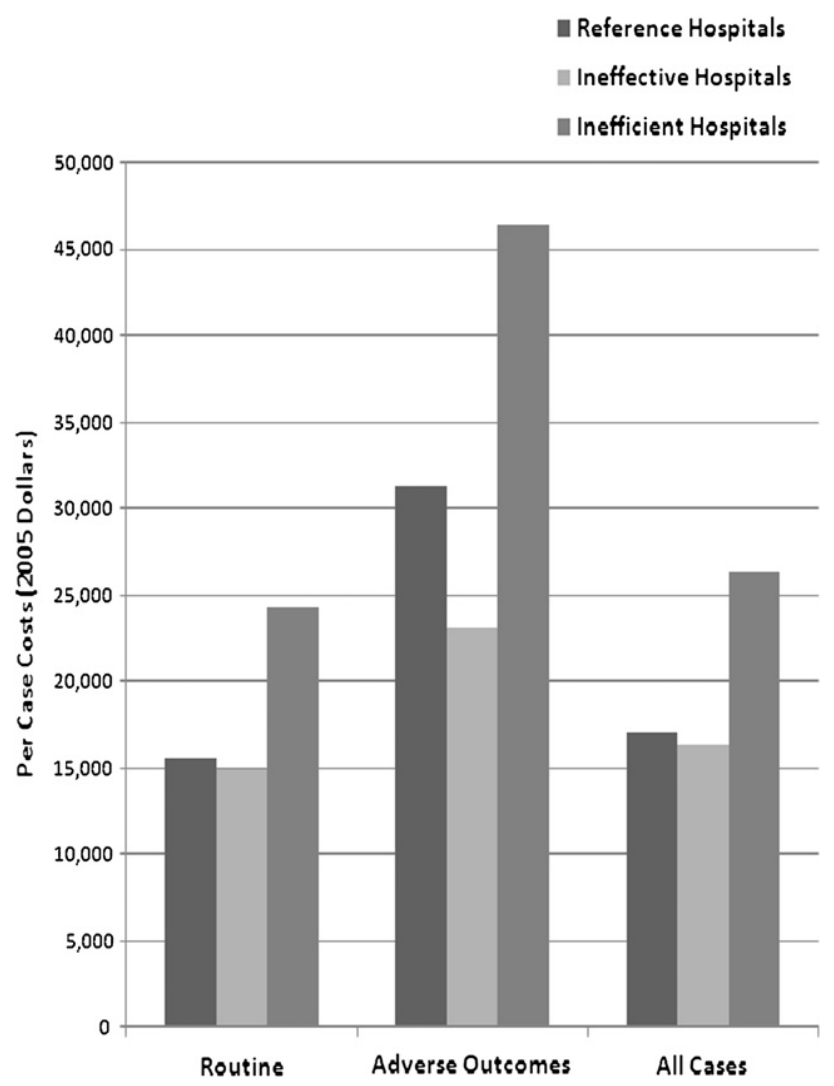

FIGURE 1. This is a graphic representation of the costs per case for ineffective, inefficient, and reference hospitals. Within each hospital group, the costs of routine cases, adverse outcome cases, and all cases together are represented.

than predicted, and inefficient hospitals had total costs that were \$15,026,461 (37.9\%) higher than predicted.

\section{DISCUSSION}

In this study, the effectiveness and efficiency of hospital care for patients undergoing elective lung resections were assessed by comparing inpatient adverse outcome rates and routine costs with the corresponding predicted values derived from the performance of a reference group of cost-effective hospitals. It is noteworthy that even when excess hospital costs associated with adverse outcomes were added to the routine costs of ineffective hospitals, the total costs at these ineffective hospitals were lower than the predicted costs derived from the performance of cost-effective hospitals. This finding is consistent with previously published analyses of elective colon resection, total joint replacement, coronary artery bypass grafting, hysterectomy, and peripheral vascular reconstruction. ${ }^{13-15}$ Thus although quality improvement initiatives will reduce excess hospital costs associated with the prevention of specific adverse outcomes, these data indicate that additional costs may be required to achieve better clinical results in

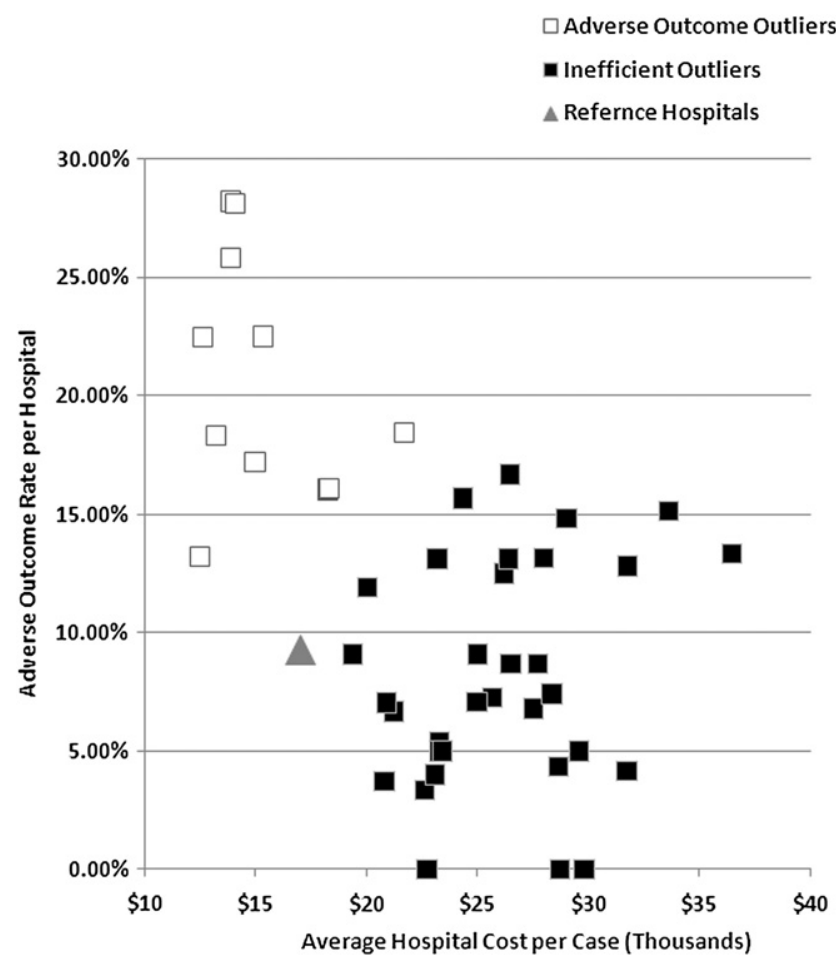

FIGURE 2. This is a scatterplot chart of adverse outcome rates plotted against average costs per case of ineffective and inefficient hospitals. The average of all reference hospitals is represented by the large triangle. These are actually observed rates and not observed minus predicted rates.

suboptimally performing hospitals. The greatest opportunity for the reduction of excess inpatient costs appears to depend primarily on reducing routine costs at inefficient hospitals.

The analytic methods used in this study are ideally suited to support episode-based, risk-adjusted bundled payments for surgical care, with quality and cost indexed to the performance of cost-effective reference hospitals. Through the use of more comprehensive data, such as may be obtained from all-payer claims databases, these methods can be expanded to incorporate the cost of professional inpatient services and postdischarge outcomes and costs. Only with such expansion can the full spectrum of costs for an inpatient episode be fully defined.

Bundled payments for entire episodes of care have been recommended by the Medicare Payment Advisory Commission. ${ }^{16}$ The Centers for Medicare and Medicaid Services have launched demonstration projects in elective joint replacement and cardiac surgery to test this method of payment. ${ }^{17}$ Episode-based payment that transfers financial responsibility for the excess cost of complications from payers to providers is a central component of the Geisinger Clinic's ProvenCare program. ${ }^{18}$ As demonstrated in this study, fair prospective prices for this type of health care warranty can be established by multiplying predicted adverse outcome rates by predicted excess costs adverse 
outcomes. ${ }^{19}$ Bundled payments that include health care warranties would enable effective, efficient hospitals and physicians to increase their net revenue and would provide strong financial incentives to coordinate care and improve clinical performance with or without actual vertical integration of hospitals and physicians. ${ }^{20}$

This study is a first effort to model the quality and cost of lung resection, and it has limitations. Hospital claims data such as those used in this study often fail to provide the clinical detail required to assess fully the risk-adjusted health care outcomes and costs. Evolving technologies such as video-assisted thoracoscopic ${ }^{21}$ and robotic techniques ${ }^{22}$ are not captured; however, they should be more easily captured in future cost and quality assessments. Clinical registries such as the Society of Thoracic Surgeons cardiac and lung cancer databases are better suited to determine preoperative risk more fully, assess unique and evolving technological interventions, and completely identify surgical complications. These databases, however, can be costly to develop and to maintain. Clinically enhanced claims databases that include present-on-admission codes to differentiate hospital-acquired complications from preoperative risk factors and numeric laboratory data from electronic data repositories have the potential to be cost-effective alternatives to costly clinical registries for monitoring risk-adjusted hospital outcomes and costs. ${ }^{23,24}$

Another limitation lies in assessment of the severity of coded complications. Generic surgical complications include more than 40 groups of ICD-9-CM diagnosis codes, and additional codes are required to identify unique complications of specific procedures (eg, bronchopleural fistula after lung resection). Many of these diagnoses include complications that can range from trivial physiologic derangements to life-threatening clinical conditions. Some may not be apparent unless specific diagnostic tests are performed. Documentation and coding of hospital-acquired complications thus may vary widely among hospitals, particularly for less serious adverse events. In this study, problems arising from inconsistencies in the diagnosis and reporting of hospital-acquired complications were minimized by limiting measured complications to those that were associated with inpatient mortality or with significantly longer than predicted postoperative stay.

In summary, this study demonstrates how analyses of risk-adjusted clinical outcomes and costs can be combined to identify reference cost-effective providers, create quality and efficiency standards derived from the performance of these cost-effective providers, and evaluate the relationship between quality and cost. It provides strong evidence that substantial reductions in hospitals costs cannot be achieved without improving the efficiency of hospital care. It presents a blueprint with more complete data sets for how bundled payments can be designed and calibrated to reward providers that deliver coordinated cost-effective care.
With the development of integrated clinically enhanced claims databases, this analytic infrastructure can serve as the basis for a reformed health care delivery system that recognizes and rewards high-quality clinical care, preserves professional autonomy, and encourages the efficient use of clinical resources.

\section{References}

1. Zhan C, Miller MR. Excess length of stay, charges, and mortality attributable to medical injuries during hospitalization. JAMA. 2003;290:1868-74.

2. Fry DE. Surgical site infections and the surgical care improvement project (SCIP): evolution of national quality measures. Surg Infect (Larchmt). 2008;9:579-84.

3. Bratzler DW, Houck PM, Richards C, Steele L, Dellinger EP, Fry DE, et al. Use of antimicrobial prophylaxis for major surgery: baseline results from the National Surgical Infection Prevention Project. Arch Surg. 2005;140:174-82.

4. Kozower BD, Sheng S, O’Brien SM, Liptay MJ, Lau CL, Jones DR, et al. STS database risk models: predictors of mortality and major morbidity for lung cancer resection. Ann Thorac Surg. 2010;90:875-83.

5. Brunelli A, Rocco G, Van Raemdonck D, Varela G, Dahan M. Lessons learned from the European thoracic surgery database: the composite performance score. Eur J Surg Oncol. 2010;36(Suppl. 1):S93-9.

6. Agency for Healthcare Research and Quality. National Inpatient Sample: Healthcare Cost and Utilization Project [Internet]. Rockville (MD): The Agency. [cited $2010 \mathrm{Jul}$ 20]. Available from: http://www.ahrq.gov/data/hcup/

7. Fry DE, Pine M, Jones BL, Meimban RJ. Adverse outcomes in surgery: redefinition of post-operative complications. Am J Surg. 2009;197:479-84.

8. Friedman B, De La Mare J, Andrews R, McKenzie DH. Practical options for estimating cost of hospital inpatient stays. J Health Care Finance. 2002;29:1-13.

9. Elixhauser A, Steiner C, Harris D, Coffey RM. Comorbidity measures for use with administrative data. Med Care. 1998;36:8-27.

10. Pursell DE. Improving population estimates with the use of dummy variables. Demography. 1970;7:87-91.

11. Wheeler DJ. Understanding variation: the key to managing chaos. 2nd ed. Knoxville, (TN): SPC Press; 2000. p. 137.

12. Lee $\mathrm{K}, \mathrm{McGreevey} \mathrm{C}$. Using control charts to assess performance measurement data. Jt Comm J Qual Improv. 2002;28:90-101.

13. Pine M, Fry DE, Jones BL, Meimban RJ, Pine G. Controlling costs without compromising quality: paying hospitals for total knee replacement. Med Care. 2010; 48:862-8.

14. Fry DE, Pine M, Jones BL, Meimban RJ. Comparative effectiveness and comparative efficiency in peripheral vascular surgery. Am J Surg. 2011;201:363-8.

15. Fry DE, Pine M, Jones BL, Meimban RJ. The impact of ineffective and inefficient care on the excess costs of elective surgical procedures. J Am Coll Surg. 2011; 212:779-86.

16. Hackbarth G, Reischauer R, Mutti A. Collective accountability for medical care-toward bundled Medicare payments. N Engl J Med. 2008;359:3-5.

17. Centers for Medicare and Medicaid Services. Medicare acute care episode demonstration (ACI). Available at: http://www.cms.hhs.gov/DemoProjectsEvalRpts/ $\mathrm{MD} /$ itemdetail.asp?filterType $=$ none $\&$ filterByDID $=-99 \&$ sortByDID $=3 \&$ sort Order $=$ descending \&itemID $=\mathrm{CMS} 1204388 \&$ intNumPerPage $=10 . \quad$ Accessed Sept 19, 2011.

18. Casale AS, Paulus RA, Selna MJ, Doll MC, Bothe AE Jr, McKinley KE, et al. "ProvenCare ${ }^{\mathrm{SM}}$ ": a provider-driven pay-for-performance program for acute episodic cardiac surgical care. Ann Surg. 2007;246:613-23.

19. Fry DE, Pine M, Jones BL, Meimban RJ. Surgical warranties to improve quality and efficiency in elective colon surgery. Arch Surg. 2010;145:647-52.

20. Fry DE, Pine M, Pine G. Virtual partnerships: aligning hospital and surgeon incentives. Am J Surg. 2010;200:105-10.

21. Farjah F, Wood DE, Mulligan MS, Krishnadasan B, Heagerty PJ, Symons RG, et al. Safety and efficacy of video-assisted versus conventional lung resection for lung cancer. J Thorac Cardiovasc Surg. 2009;137:1415-21.

22. Veronesi G, Galetta D, Maisonneuve P, Melfi F, Schmid RA, Borri A, et al. Fourarm robotic lobectomy for the treatment of early-stage lung cancer. J Thorac Cardiovasc Surg. 2010;140:19-25.

23. Pine M, Jordan HS, Elixhauser A, Fry DE, Hoaglin DC, Jones B, et al. Enhancement of claims data to improve risk adjustment of hospital mortality. JAMA. 2007;297:71-6.

24. Fry DE, Pine M, Jordan HS, Elixhauser A, Hoaglin DC, Jones B, et al. Combining administrative and clinical data to stratify surgical risk. Ann Surg. 2007;246: 875-85. 